\title{
Escuelas normales de mujeres en Colombia (1903-1914)
}

\author{
Normal schools of women in Colombia (1903-1914)
}

Escolas normais femininas na Colômbia (1903-1914)

Yesica Paola Montes Geles $^{1}$ - Nilce Vieira CAMpos Ferreira ${ }^{2}$

\section{Resumen}

El objetivo es analizar las escuelas normales teniendo como fuentes las disposiciones de la Ley Orgánica de 1903 y el Decreto 491 de 1904, lo cual hace parte de una investigación en desarrollo, cuyo propósito inicial es estudiar la Escuela Normal de Institutoras de Bolívar en el período de 1903 hasta 1930, con el análisis de fuentes documentales y registros históricos de la época. Exponemos una visión general de las leyes orgánicas en la historia de la educación colombiana partiendo de la Ley Orgánica de 1903, segunda ley que reforma la Instrucción Pública colombiana. Procuramos responder: cuáles son las mudanzas en la organización de las Escuelas Normales femeninas en el período de 1903 a 1914? La reforma defendida por la Ley Orgánica buscaba principalmente estimular la formación de profesores y promover las escuelas normales para hombres y mujeres con el objetivo de alcanzar un índice educativo más elevado en Colombia.

Palabras clave: Ley Orgánica. Decreto 491. Escuelas Normales de Mujeres. Colombia.

\footnotetext{
${ }^{1}$ Mestre em Educação pela Universidade Federal de Mato Grosso. Professora adjunta da Corporación Universitaria del Caribe/Colombia (Campus Sincelejo). Pesquisadora do Grupo de Pesquisa e Estudos em História da Educação, Instituições e Gênero, do Grupo de Pesquisa Educación, Universidad y Sociedade da Universidad de Cartagena e da Rede Mulheres pela Equidade de Gênero. E-mail: yesica.montes.g55@gmail.com

${ }^{2}$ Doutora em Educação pela Universidade Federal de Uberlândia. Professora Adjunta da Universidade Federal de Mato Grosso (UFTM). Coordenadora do Centro Memória Viva do Instituto de Educação da UFMT. Coordenadora do Grupo de Investigação e Estudos em História da Educação, Instituições e Gênero. E-mail: nilcevieiraufmt@gmail.com
} 


\begin{abstract}
This study aims to analyze the normal schools having as sources the rules of the Organic Law of 1903 and the decree 491 from 1904, which is part of a developing research, whose initial purpose is to study La Escuela Normal de Institutoras de Bolivar during the period from 1903 to 1930, with the analysis of the documental sources and historical records of the age. It is presented a general overview of the organic laws in the history of Colombian education starting from the Organic Law of 1903, second law that reforms the Colombian Public Instruction. It is tried to answer: What are the changes in the organization of feminine normal schools during the period from 1903 to 1914? The reform defended by the Organic Law sought mainly to stimulate the teachers training and to promote the normal schools for men and women with the objective of achieving a higher educational index in Colombia.
\end{abstract}

Keywords: Organic Law, Decree 491, Normal School for Women, Colombia.

\title{
Resumo
}

Objetivamos analisar as escolas normais tendo como fontes as disposições da Lei Orgânica de 1903 e do Decreto 491 de 1904, cujo foco é estudar a Escola Normal do Instituto de Bolívar no período de 1903 a 1930, com a análise de fontes documentais e registros históricos da época. Expomos uma visão geral das leis orgânicas na história da educação colombiana com base na Lei Orgânica de 1903, a segunda lei que reforma a Instrução Pública Colombiana. Procuramos responder: quais são as mudanças na organização das Escolas Normais Femininas no período de 1903 a 1914? Concluímos que a reforma preconizada pela Lei Orgânica buscou principalmente estimular a formação de professores e promover escolas normais para homens e mulheres com o objetivo de alcançar um índice educacional mais alto na Colômbia.

Palavras-chave: Lei Orgânica. Decreto 491, Escola Normal Feminina. Colômbia. 


\section{Introducción}

La historia de las Escuelas Normales Femeninas de Colombia ha estado marcada por el recorrido social, económico y político del país y de las leyes que fundamentaron la Instrucción Pública. En Colombia, solamente han existido tres leyes generales de educación. La primera de ellas, bajo el mandato del liberalismo ${ }^{3}$, fue el Decreto Orgánico de Instrucción Pública de 1870 (DOIP) que intenta ampliar el concepto de educación integral y modificar los conceptos de enseñanza produciendo grandes avances en la pedagogía de la época. La segunda es la Ley Orgánica de 1903 centrada en una educación católica y conservadora ${ }^{4}$, donde el estado no podía intervenir en las instituciones privadas, pero la iglesia tenía el derecho de hacerlo en las oficiales (BOCANEGRA, 2008, p. 113). Por último, la vigente Ley 115 de 1994 o Ley General de Educación fundamentada en la Constitución Política de Colombia de 1991 y la Asamblea Nacional Constituyente del mismo año.

Estas leyes fueron el resultado de triunfos de proyectos políticos, que llevaron a la educación colombiana a experimentar un cúmulo de contradicciones y disputas ideologías (BOCANEGRA, 2008), donde las Escuelas Normales, tanto de varones como femeninas, en relación a la formación de maestros fueron centros de cambios y reformas continuas. Estos cambios marcaron la historia de las Escuelas Normales en el país y trajeron consigo mudanzas en las prácticas educativas, lo que nos lleva a pensar que es de vital importancia en el campo de la historiográfica realizar investigaciones en las cuales se busque entender la realidad histórica de las Escuelas Normales colombianas, puesto que son las responsables por la formación de profesores desde 1821.

En este trabajo analizamos la Ley Orgánica de Educación de 1903 y el Decreto 491 de 1904 que la reglamenta, para entender, desde la Ley, los cambios que han tenido las Escuelas Normales Femeninas desde su creación en 1874 y el lugar que ocuparon estás en las disposiciones legales analizadas.

Sin embargo, es pertinente resaltar que la Instrucción Pública en Colombia desde la creación de la Ley Orgánica se solidifica y reafirma en muchas otras leyes, entre ellas, la expedición de la Ley 38 de 1914 que ordena la creación del Instituto Agrícola Nacional, la Ley 31 de 1917 de estipula la enseñanza industrial en todo el territorio colombiano y la Ley 37 de 1923 que mantiene vigente el Instituto de Comercio. Tres leyes que insertan a la enseñanza los principios de las tres fuentes de riqueza pública: el comercio, la agricultura y la industria, que hacen de la Instrucción pública colombiana una legislación sintética, sustanciosa y orgánica (URIBE, 1927, p. 9).

Para el análisis tomamos como texto fundamental la Revista de Instrucción Pública. Disposiciones Vigentes de 1927, donde se hallan la Ley Orgánica de 1903, el Decreto 491 de 1904 y las demás leyes que rigen la Instrucción Pública desde el periodo conservador. En primer lugar, se realiza un esbozo general acerca la Ley Orgánica, su creación e importancia para la Instrucción Pública. En segundo lugar, se detallan los fundamentos principales de la Ley, dando una mirada más profunda hacia los reglamentos que competen las Escuelas Normales. En tercer

\footnotetext{
${ }^{3}$ El liberalismo es un movimiento político que fundamenta sus bases en la ilustración kantiana, promueve el Estado laico y está en contra de las monarquías absolutas.

${ }^{4}$ El conservadurismo nació en oposición a las políticas liberales y se caracteriza por defender la participación de la iglesia en el Estado y en la sociedad, el respeto por la autoridades y por la jerarquía.
} 
lugar, se muestras las normas que, dentro de las disposiciones generales sobre la Instrucción Pública, rigen y fundamentan las Escuelas Normales del país. Y, por último, se analiza qué lugar ocupan las Escuelas Normales Femeninas en la Ley para entender cómo se organizaba la educación femenina en una época de hegemonía conservadora 5 .

\section{¿Por qué nace la Ley Orgánica de Instrucción Pública en 1903?}

La Ley 39 de 26 de octubre de 1903 o segunda Ley Orgánica de Instrucción Pública de Colombia, también es conocida históricamente con el nombre de Ley Uribe, en honor al entonces ministro de Instrucción Pública, quien la reglamenta y defiende. Esta Ley, regulada en el año de 1904 por el Decreto 491, plantea un nuevo sistema de educación en Colombia en concordancia con las particularidades y necesidades del país e integra las normas sobre los museos, bibliotecas y archivos públicos. Ríos Beltrán (2004, p. 79) se refiere a ella como el punto de partida de toda reforma educativa en la primera mitad del siglo XX y argumenta:

Con esta ley se comienza a plantear la necesidad de hacer cambios, tomar nuevos rumbos y orientaciones en la empresa de instruir la niñez y la juventud colombiana. De esta manera se introducen nuevos saberes y métodos para la formación de maestros y las nuevas generaciones perfilándose así la formación de un sujeto productivo, útil, con iniciativa individual y amante del trabajo frente al hombre pasivo, funcionalista y ocioso del siglo XIX (RIOS, 2004, p. 79).

La nueva reglamentación de la educación colombiana históricamente es considerada el fundamento jurídico del sistema educativo colombiano (MOLANO y VERA) puesto que, frente al Decreto Orgánico de Instrucción Pública de 1870 que decretaba la obligatoriedad de enseñanza elemental, la Ley 39 aprueba la educación elemental gratuita y no obligatoria, promoviendo su universalización. Esto, marca el antes y el después del sistema educativo colombiano.

La Ley Orgánica de Instrucción Pública de 1903 en relación al anterior de Decreto Orgánico de 1870 creado en concordancia con la Constitución Política de Rionegro de 1853, le otorga valor a la educación como proceso para formar una nación moderna, por lo que busca redefinir la formación de los maestros, a través de nuevos métodos pedagógicos implementados en las Escuelas Normales, así como el fortalecimiento y ampliación de las mismas en todo el territorio nacional.

Antonio José Uribe (1927, p. 4) afirma que la Ley se expide luego de realizar un balance detallado de los 5 momentos esenciales por los que pasa la legislación de la instrucción pública colombiana, desde la independencia hasta 1903. Vistos a grande escala, los periodos a los que se refiere Uribe son:

\footnotetext{
${ }^{5}$ Es el período entre 1886 y 1930, en el cual el Partido Político Conservador Colombiano toma el poder por más de 44 años y la iglesia es vista como el centro del Estado y de la sociedad.
} 
1. Un primer momento que inicia con la expedición de la Ley de 18 de marzo de 1826, donde se reglamenta la educación pública para los ciudadanos, teniendo en cuenta sus talentos y sus inclinaciones; así como la instrucción general en escuelas de enseñanza primaria y elemental, y la instrucción especial, en universidades departamentales y especiales; y que culmina con la ley 7 de 21 de mayo de 1842.

2. El segundo momento comienza con la ley 7 de 21 de mayo de 1842, donde se autorizan las reformas para las universidades y para los planes de Instrucción Pública.

3. Un tercer momento marcado por lo dispuesto en la Ley 2 de 15 de mayo de 1850, la cual otorga libertad a la enseñanza de todos los ramos de las ciencias, las artes y las letras, abole las universidades y suprime el grado de bachiller.

4. El cuarto período inicia con la Ley 66 de 16 de septiembre de 1867 que reglamenta la creación de la Universidad Nacional de Colombia con asiento en la ciudad de Bogotá, dándose la apertura de seis institutos o escuelas especiales: medicina; literatura y filosofía; ciencias naturales; artes y oficios; derecho e ingeniería.

5. El último momento se centra en las disposiciones de la Constitución Política de Colombia de 1886, donde surge una nueva reforma de instrucción popular, marcada por una enseñanza gratuita y el restablecimiento de la enseñanza clásica en la Facultad de Filosofía y Letras.

La creación de la Ley Orgánica, en el mandato del presidente José Manuel Marroquín, fue, en su momento, la herramienta legislativa más útil para la regulación y direccionamiento de los nuevos procesos educativos, puesto que en ella se construye un sistema escolar y un sistema universitario, y a la vez, se implementa la clasificación de la educación formal. De igual manera, se establecen normas para el fortalecimiento de las escuelas normales en todo el país.

\section{Fundamentos de La Ley Orgánica de 1903}

Unos de los cambios más importantes de la Ley Orgánica fue la imposición de la religión católica como eje de la educación colombiana, aboliendo la libertad de cultos proclamada por los liberales de los años 70 en el Decreto Orgánico de 1870. En relación a este último, Jaramillo expone:

Ambos principios, el de la obligatoriedad de la enseñanza elemental y el de la neutralidad religiosa, dieron lugar a un largo y complejo conflicto con la Iglesia y con amplios sectores de la opinión pública. Se veía en ellos una negación del principio de la libertad de enseñanza que garantizaba la constitución y una incoherencia con los sentimientos católicos de la mayoría de la población nacional (JARAMILLO, 1980).

Según, Jaime Jaramillo (1980), estos dos elementos fueron los más innovadores y polémicos dentro del Decreto Orgánico de 1870, especialmente el reglamentar la libertad de religión dentro de una sociedad mayoritariamente católica. Pero, en el 1886, aparece la hegemonía conservadora y se estipula la religión católica como base de la Instrucción Pública, tal como se manifiesta en el Artículo $1^{\circ}$ de la Ley Orgánica de 1903: "La instrucción Pública en Colombia será organizada y dirigida por concordancia con la Religión Católica”(COLOMBIA, 1903). 
Otro importante cambio presente en el Ley 39 de 1903 es ofrecer una educación libre en todos los grados, es decir, se abole el sentido de obligatoriedad de la educación pre sente en el Decreto de 1870. Esto, porque la obligatoriedad exigía a los padres de familia que enviaran a sus hijos a la escuela, imponiendo sanciones a los que incumplieran. Es claro, que la propuesta de una educación igualitaria para todos era favorable, sin embargo, en las familias rurales, donde los niños se dedican a trabajar el campo junto a sus padres, la educación y su obligatoriedad era vista como un problema, lo cual ponía en conflicto la relación del pueblo y el gobierno.

De igual forma, la Instrucción Pública en el Decreto de 1870 que era dividida en tres ramos (enseñanza, inspección y administración) y en la Ley Orgánica es dividida en cuatro ciclos: primario, secundario, industrial y profesional, con el objetivo de formar una educación completa. A partir de aquí, la educación adquiere un papel importante para el desarrollo de la economía, pues se organiza una educación técnica focalizada a enseñar para la productividad y desarrollo de las riquezas del país.

La instrucción primaria está cargo de los Gobiernos de los Departamentos y será inspeccionada por el Poder Nacional. Los docentes enfocarán la enseñanza al ejercicio de la ciudadanía, la industria, el comercio, la vida religiosa para formar hombres nobles y sabios. Por otro lado, la instrucción secundaria es inspeccionada por el Poder Ejecutivo y la Nación se hace cargo de ella y la educación es esencialmente técnica y clásica. La primera guiada al estudio de los idiomas vivos, las ciencias físicas y las matemáticas que preparen al ciudadano para una carrera industrial. La segunda se encarga de preparar profesores liberales, por lo que en ella se estudian Letras y Filosofía.

La instrucción profesional, por su parte, está enfoca la educación hacia la teoría y práctica del conocimiento, de una forma exigente y estricta, con el fin de educar a los mejores doctores en ciencias, derecho e ingeniería. Los institutos de minería y agricultura atenderán de modo científico la explotación de los recursos naturales del país y los demás cuerpos científicos deben dedicarse al estudio de problemas patrios.

Por otro lado, las escuelas de música y Bellas Artes continúan bajo el gobierno de la Escuela de Música y Bellas Artes; al igual que las bibliotecas y museos nacionales, los cuales siguen bajo la reglamentación del Ministerio de Instrucción Pública. En los primeros 5 artículos de La Ley Orgánica, se hace evidente, la asignación de obligaciones de Instrucción Pública a los diferentes entes del país. Por lo que Ospina (2013) afirma:

Esta ley buscaba, frente al decreto Orgánico de Instrucción Pública de 1870 primera Ley Orgánica de Educación, fortalecer el control estatal sobre toda la estructura educativa, asignando responsabilidades a los departamentos, en términos administrativos (dirección, protección, vigilancia, control, nombramiento y evaluación de maestros), y a los municipios, en términos operativos (sostenimiento, dotación y mantenimiento de instalaciones educativas); se establecía, igualmente, que la instrucción primaria que fuera costeada con fondos públicos pasaba a ser gratuita y no obligatoria, al mismo tiempo que se promovía su universalización. 
Con todo esto, se establece la descentralización de la educación en Colombia para que las entidades políticas puedan atender a la instrucción popular y si es posible se cree la autonomía en los institutos de enseñanza. Claro está, siempre manteniendo una inspección constante a cargo de juntas que regulen el sistema público a nivel municipal, departamental y nacional. Es así como, confiando plenamente en lo dispuesto en la Ley 39 de 1903, Antonio José Uribe expone:

La ley orgánica a la que nos referimos estableció la armonía entre los derechos de los individuos, las familias de las corporaciones, del estado y de la Iglesia, así como el conveniente consorcio entre las actividades del municipio, del departamento y de la Nación para trabajar de consuno en la obra múltiple y fecunda de la instrucción y educación del pueblo (URIBE, 1927, p. 8).

Para el entonces Ministro de Instrucción Pública, la Ley Orgánica de 1903 era el instrumento facilitador de relaciones entre las entidades del país. Así como también, era la creadora, gracias a sus disposiciones, del equilibrio entre los entes que conforman la sociedad colombiana de principios del siglo XX. En fin, La Ley Orgánica, tal como estaba organizada, era el elemento primordial para alcanzar un mayor nivel educativo en Colombia. Ello, porque para su creación, en palabras de Uribe, se analizó la trayectoria y método educativos de países con mayores avances en ámbito de la Instrucción Pública.

En el año de 1903, La Instrucción Pública era la base más sólida del cambio y el progreso en el país. Todas las expectativas estaban puestas en ella y en su buena ejecución por parte de los entes públicos. Tanto así que se consideraba que no era necesaria una nueva Instrucción Pública, sino el adecuado seguimiento de las disposiciones presentes en ella. Desde esa perspectiva, el porvenir de la Patria, no era una labor constituyente y legislativa, sino administrativa, ejecutiva y docente (URIBE, 1927, p. 12). Fue tan grande el apoyo a esta nueva reforma educativa que la Ley orgánica de Instrucción Pública de 1903 estuvo vigente por más de 90 años hasta ser sustituida por la Ley General de Educación en 1994.

\section{Surgimiento de las Escuelas Normales de Colombia}

Histórica y legalmente, la creación de las Escuelas Normales en Colombia se estipula en la Ley de 6 de agosto de 1821. Fue Fray Sebastián Mora quien dirige la primera escuela de enseñanza mutua con método lancasteriano o monitorial en Colombia, donde se instruía al profesor para enseñar lectura, escritura, aritmética, buenos modales, historia, geografía y religión a un gran número de estudiantes, lo que le ahorraba a la nueva República recursos económicos que en la época eran escasos (PARRA, 2013, p. 11).

Sin embargo, los conocimientos adquiridos por parte de los profesores formados en las Escuelas Normales Lancasterianas eran casi iguales a los adquiridos por los alumnos de las escuelas primarias, lo único que los diferenciaba era el método de enseñanza, por lo que las Escuelas Normales eran centros de reproducción de enseñanza mutua, donde los profesores estaban lejos de cumplir con el papel de educadores. Fue con la Ley de 26 de junio de 1842 que las Escuelas Normales adquirieron una relación directa con la formación de profesores. (ZULUAGA, 1996, p. 275) 
Sin lugar a dudas, la escuela normal como institución para formar maestros y promover un saber sobre la enseñanza se inicia con las reformas de Ospina. En octubre de 1844 se inauguró la escuela normal del distrito de Bogotá en un edificio construido de acuerdo con las normas del método lancasteriano. El método dejó de ser el único conocimiento pedagógico de los maestros (ZULUAGA, 1996, p. 278).

Según Zuluaga (1996) es con la reforma de Ospina que las Escuelas Normales adquieren el sentido de formadoras de maestros. Su función no se limita a la reproducción de métodos de enseñanza, sino que se amplía hacia la pedagogía. Se incrementa la inspección del saber y de las habilidades del maestro para la enseñanza, creando programas de pedagogía y dándole identidad como institución al separarla de la escuela parroquial.

Ahora bien, es en el Decreto Orgánico de 1870 que se reglamenta la fundación de la Escuela Normal Central en la ciudad de Bogotá para instruir maestros encargados de la enseñanza en las Escuelas Normales de todo el país y, una Escuela Normal por cada capital de Estado ${ }^{6}$ que forme docentes para las escuelas elementares y superiores. De igual forma, se establece la creación de la revista Escuela Normal, órgano oficial de publicidad de todos los actos del poder ejecutivo y de los asuntos relativos a la enseñanza. En total, dos elementos esenciales que para Jaramillo (1980) constituyen uno de los aspectos más productivos del decreto.

Aunque en el Decreto Orgánico de Instrucción Pública de 1870 no se encuentra estipulada la creación de las Escuelas Normales Femeninas, es en el periodo Liberal Radical que nacen estas instituciones escolares. Ello, porque la educación femenina llegó a considerarse uno de los principales aspectos para el desarrollo de la Instrucción Pública, puesto que el gobierno de la época buscaba estar a la altura de los países de Europa, preocupados por la educación de la mujer, así como también, la escases de maestras para la instrucción primaria exigía tomar medidas para atender la educación de niñas.

Así, en el gobierno del presidente Santiago Pérez se reglamenta el Decreto Ejecutivo Federal $n^{\circ} 356$ de 27 de agosto de 1874 que establece en todo el país la creación de las Escuelas Normales de Institutoras, cuyo objetivo fundamental se estipula en su artículo 3:

formar maestras idóneas para rejentar las escuelas primarias de su sexo en los Estados respectivos. En consecuencia, anexa a cada una habrá una Escuelas primaria elemental, donde se ensayarán, para la instrucción de las alumnas maestras, los métodos de enseñanza.

A partir de aquí se crearon las Escuelas Normales de Institutoras, una en cada capital de los Estados Soberanos del país, donde se formaban las mujeres que serían las encargadas de la Instrucción primaria de niñas. En total, para el año 1878 existían en Colombia aproximadamente diez Escuelas Normales de Institutoras, ocho de ellas creadas bajo el Decreto Ejecutivo Federal n ${ }^{\circ} 356$ de 1874 (BÁEZ, 2005, p. 442).

\footnotetext{
${ }^{6}$ En 1863, con la proclamación de la Constitución de Rionegro, lanzada por los Liberales Radicales, Colombia adoptó el nombre de Estados Unidos de Colombia y estuvo conformada como una federación de Estados Soberanos casi independientes. Sin embargo, en 1886, cuando los conservadores llegan al poder, surgió una nueva constitución que acaba con el federalismo y organiza el centralismo, en el cual es país depende administrativa y políticamente de una región capital (Bogotá), que tiene departamentos administrativos. A partir de esta constitución, el país es llamado República de Colombia.
} 


\section{Escuelas Normales en la Ley Orgánica de 1903 y en el Decreto 491 de 1904}

La Ley Orgánica de 1903, solo ocupa tres artículos para exponer los reglamentos relacionados con las Escuelas Normales. El primero de ellos, el artículo $13^{\circ}$, establece la creación de dos Escuelas Normales en cada una de las ciudades capitales del país, siendo una para hombres y otra para mujeres. Cada institución deberá ser vigilada por la entidad departamental, pero será costeada por la Nación. Así mismo, la Escuela Normal para hombres, dentro de su equipo de empleados, tendrá un Prefecto General de Estudio que no es decretado para la escuela Normal de mujeres.

El principal objetivo con las Escuelas Normales expuesto en La Ley Orgánica de 1903 era formar maestros prácticos y pedagógicos, capaces de enseñar de una forma fácil e dinámica. Por ello, además de formar en la moral y la religiosidad, los docentes de las Escuelas Normales, según el artículo $15^{\circ}$, debían fomentar el estudio del comercio, la industria y la agricultura, especialmente en los estudiantes de secundaria. En palabras de Uribe es:

Las Escuelas Normales tiene doble misión: la primera es completar la instrucción general de los futuros institutores: la segunda es su función principal y específica, darles una instrucción profesional sólida. Tres años bastarían para conseguir este último objeto, siempre que la instrucción general que sirve de base a esta formación profesional y que puede adquirir en los cursos completos de la instrucción primaria, sea bastante extensa (URIBE, 1927, p. 25).

Claro es, que la educación en la Escuelas Normales no era igual a la impartida en otros institutos o escuelas. Por lo tanto, se exige la ejecución de ejercicios de investigación vistos en espacios de talleres, seminarios y laboratorios para insertar al estudiante en el campo de la documentación y despertar en él la curiosidad intelectual. No es posible, dentro de las Escuelas Normales que solo se instruyan en los saberes generales, se deben formar educadores a través de saberes específicos y prácticos (URIBE, 1927, p. 25).

Con respecto a los estudiantes, se plantea, en el artículo $14^{\circ}$, que pueden ser becados por la Nación o el departamento ${ }^{7}$ y estas becas serán proporcionales al número de habitantes de este último. Tales becas eran incentivos del gobierno para que los jóvenes, especialmente los hombres, acogieran la docencia primaria como una carrera profesional, puesto que para ellos los estudios normalistas eran extensos y difíciles comparados con el status que ganaban siendo profesores de primaria (HELG, 1987, p.88).

Es en el Decreto 491 de 1904 que aparece un capítulo que atiende las disposiciones de las Escuelas Normales. En el capítulo I del título XII sobre Instrucción Secundaria se presenta detalladamente los reglamentos de las escuelas formadoras de maestros. El primer reglamento es la creación de Escuelas Anexas destinadas a la educación en su ciclo primario y al ejercicio práctico de los métodos de enseñanza adquiridos en las Escuelas Normales, es decir, los

\footnotetext{
${ }^{7}$ En Colombia, con la organización del país como República, los Estados Soberanos, en su mayoría, se convierten en Departamentos, siendo estos subdivisiones administrativas del país.
} 
docentes en formación realizan sus prácticas pedagógicas dentro de la Escuela Anexa. De igual forma, expone que los profesores de estas escuelas deben ser del mismo sexo que el de los alumnos. Así lo resume Saénz:

La Ley Uribe de 1903 ordenó que en cada departamento se abriera una escuela normal para varones y otra para mujeres, las cuales contarían con una escuela primaria anexa para los ejercicios prácticos de los métodos de enseñanza. Esta ley se fundamentó en dos principios que marcarían el devenir del saber pedagógico y la educación pública hasta mediados del siglo: el énfasis en la dimensión metodológica de la pedagogía y la finalidad educativa de progreso económico (SAÉNZ, 1997, 160).

Por otro lado, reglamenta las disciplinas a desarrollar en los cuatro años de formación profesoral. Estas asignaturas son generalizadas tanto en las Escuelas de Varones como en las Escuelas de Mujeres con algunas diferencias temáticas. En la educación masculina se orienta una formación hacia las nociones de las ciencias naturales, la agricultura, la horticultura y la arboricultura, junto a disciplinas de álgebra, ecuaciones, geometría plana y del espacio. Por su parte, en la educación femenina, aunque compartía algunas disciplinas con la educación masculina como religión, moral, idioma nacional, pedagogía, metodología, centraba la formación hacia la economía doméstica, el dibujo, las formas geométricas, el trabajo de aguja y de corte. Con respecto a ello, Uribe afirma:

El decreto 491 determinó el número de cursos que deben dictarse en las escuelas Normales, y él está en armonía con lo que sobre el particular se práctica en los mejores establecimientos de esta clase en el extranjero. Lo que importa es hacer una buena distribución de aquellas materias, y, en general, determinar bien los horarios, el programa de cada curso y que el Ministerio imparta atinadas distribuciones sobre la materia como han de ser todas y cada una de aquellas enseñanzas (URIBE, 1927, p. 26).

Es evidente que, tanto en la Ley Orgánica como en el Decreto 491, se le otorga valor fundamental a la formación de maestros. Ello, a través de la constitución de normas que aumentan la creación de Escuelas Normales, el mejoramiento de su infraestructura, la formación de maestros ligada hacia una pedagogía práctica. Por lo que es válido afirmar que la reforma de la Instrucción Pública de 1903 está centrada en la pedagogía activa, las escuelas primarias y la formación de maestros, vistos estos últimos como el pilar para el futuro de la nación (SAÉNZ, 1996, p. 154). Aunque siempre regida por las doctrinas católicas. De ahí que toda institución educativa estuviera orientada hacia el catolicismo y por ende, tanto los maestros como los estudiantes, debían regirse por las normas de la iglesia.

Muestra de ello, es el artículo $5^{\circ}$ del Decreto 1244 de 21 de octubre de 1905 sobre algunas disposiciones sobre Escuelas Normales, que renueva la continuidad del contrato con los Hermanos Cristianos con respecto a la Escuela Normal Superior de varones y la Escuela 
Normal de Cundinamarca, haciendo evidente la fuerza de la religión católica en la educación. Con relación a esto, Saénz afirma:

A partir de 1904 se incrementaron los establecimientos normalistas oficiales y laicos. Pero buena parte de la formación de maestros permaneció en manos de instituciones católicas o privadas. Entre estas la principal normal y única de carácter nacional, la Escuela Normal Central de Institutores de Bogotá dirigida por los Hermanos Cristianos y la Escuela Normal de Varones de los Hermanos Maristas (SAÉNZ, 1996, 160).

\section{El lugar de las Escuelas Normales de Mujeres dentro de la Ley Orgánica y el Decreto 491}

Las Escuelas Normales de Mujeres nacen a mediados del siglo XIX con el objetivo de formar maestras para la enseñanza primaria en los institutos de niñas. A partir de aquí la mujer fue considerada como agente fundamental para la formación y la educación. Su lugar en el campo de la Instrucción pública fue tomando poder, mas siempre es vista como educadora para las actividades domésticas. Sin embargo, fue una oportunidad para que las mujeres ingresaran a una carrera profesional, adquiriendo así un lugar respetado dentro de la sociedad.

La profesión de maestra pasó a ser reconocida como una actividad propia para el bello sexo, dada la analogía que habían establecido entre "la naturaleza femenina" y la educación formal de las niñas y las jóvenes. Para la sociedad, las maestras lograban conjugar el sentido de ser madre con la capacidad de entrega al otro; existía la creencia de la supuesta capacidad innata de las mujeres para las tareas educativas, para el cuidado moral y material de la niñez (HERNÁNDEZ, 2012, p. 250).

En el año 1903, cuando se crea la segunda Ley Orgánica de Instrucción Pública, la mujer sigue ocupando el lugar de educadora de niñas, ahora regida por los cánones de la religión católica, lo que para Hernández (2011, p. 105) constituía en formar maestras que reforzaran la cultura católica del país y la imagen de la mujer obediente.

Ahora bien, si analizamos las reglamentaciones concernientes a las Escuelas Normales de Mujeres encontramos que dentro de la Ley Orgánica de 1903, los tres artículos que reglamentan las escuelas normales en el país, solo se hace mención de las Escuelas Normales de Mujeres al referirse que debe existir una en cada Estado.

De igual manera, el Decreto 491 de 1904, el cual reglamenta a la Ley Orgánica de 1903, las normas que rigen las Escuelas Normales de Mujeres son limitadas y escasas. De los 9 artículos que conforman las disposiciones sobre las Escuelas Normales, dos de ellos se refieren a las Escuelas Normales de Mujeres, donde se estipulan las normas sobre asignaturas y los empleados que harán parte de ellas, siempre en relación a las Escuelas Normales de Varones. El artículo $15^{\circ}$, por ejemplo, detalla las áreas de conocimiento que deben ser enseñadas, dejando en evidencia que la educación de las Escuelas Normales de Mujeres se direcciona hacia la preparación para la vida doméstica. 
Aunque, la educación en las Escuelas Normales de Mujeres estaba ligada aún con las actividades domésticas, la creación y fortalecimiento de estas instituciones, a través de leyes, permitió que las mujeres colombianas adoptaran una posición de prestigio y reconocimiento social. Así mismo, se le posibilita desempeñarse en una labor distinta a la del hogar por ser consideradas seres honorables y horados,

era como si las mujeres hubieran nacido para ser maestras, una interpretación que no permitía evidenciar el origen histórico-social de las vocaciones. Sin embargo, tanto la formación como la dedicación de algunas mujeres al magisterio, fueron de vital importancia para el futuro de la acción en lo público de la población femenina, en cuanto ampliaron el espectro existencial, en este caso, de las colombianas. El magisterio, se convirtió en una actividad a través de la cual algunas jóvenes de Pasto y de la región sur del país lograron dar un paso para superar la marginalidad social en la que permanecían siendo ya las postrimerías del siglo XIX (HERNÁNDEZ, 2012, p. 250).

La mujer en la educación era vista como educadora por vocación. Una persona apta para enseñar a las niñas los fundamentos básicos para la acción femenina en una sociedad conservadora, por lo que la profesora en formación debía ser una mujer responsable, culta, católica, es decir, con un comportamiento moral impecable y, al mismo tiempo, con la fuerza necesaria para asumir la responsabilidad de la Instrucción Pública de las niñas.

\section{Consideraciones finales}

El análisis exploratorio de la Ley Orgánica de 26 de octubre de 1903y del Decreto 491 de 3 de junio de 1904, nos permitió reconocer que la Instrucción Pública en Colombia, a partir de 1903, otorgó un papel fundamental a las Escuelas Normales en relación a la educación primaria y la formación de profesores. Por esto, las Escuelas normales se constituyeron como el centro de la educación colombiana de la época, principalmente porque por medio de ellas se formaban los futuros profesores para la instrucción primaria de todo el país. Claro está que se trataba de una educación centralizada y basada en los principios del catolicismo.

Estudiar las leyes que reglamentaron la creación y organización de las Escuelas Normales posibilitó el entendimiento del contexto político y social en el cual fueron construidas y el reconocimiento de las directrices que las regulaban en el periodo de estudio. Las leyes analizadas, como la Ley Orgánica y el Decreto 491, dieron luces para entender aspectos fundamentales sobre las Escuelas Normales de Colombia. El primero de ellos fue la reglamentación para construir Escuelas Normales tanto para hombres como para mujeres. El segundo, el fortalecimiento de esas escuelas como centro de formación de maestros. El tercero, la constitución de las Escuelas Normales para la enseñanza católica en una sociedad conservadora.

Con relación a las Escuelas Normales de Mujeres destacamos el avance de estas al imponerse la creación de una en cada uno de los Estados Soberanos del país y la valorización de la mujer en el campo de la educación, donde se les otorga un lugar de prestigio en la sociedad. Sin embrago, es evidente que en las leyes analizadas el porcentaje de reglamentos que tratan sobre las Escuelas Normales Femeninas es menor en comparación con aquellos que abordan escuelas normales masculinas, aspecto que es indispensable analizar con mayor profundidad. 
Las Escuelas Normales, en nuestro caso las Escuelas Normales de Mujeres, merecen un estudio más detallado en la historia de educación colombiana para logar entender la realidad histórica que dentro de ellas se guarda. Así, el análisis de las leyes que las regularon en el periodo de estudio ayuda para la comprensión del pasado que las conformó y nos permite comprender el valor de ellas dentro de la sociedad, el lugar que les fue permitido a las mujeres en la educación del país y las transformaciones por las cuales pasó la educación femenina colombiana.

Por las razones expuestas, consideramos que el campo historiográfico que aborda los estudios sobre las Escuelas Normales Femeninas es de gran relevancia y merece ser abordado a partir de diferentes perspectivas teóricas y empíricas que nos posibiliten visualizar su trayectoria.

\section{Referencias}

BÁEZ, Miryam. Las escuelas normales colombianas y la formación de maestros en el siglo XIX. Revista científica EccoS. Sao Paulo. V. 7, N. 2, julho-dezembro de 2005. Disponible en: http://www.uninove.br/PDFs/Publicacoes/eccos/eccos_v7n2/eccosv7n2_2j04.pdf. Acceso en: 3 de mayo de 2015.

BOCANEGRA, Henry. Políticas educativas, condición social del Magisterio Colombiano y su constitución como organización sindical y actor político. Revista Diálogos de Saberes, Bogotá. N. 28, enero-junio de 2008. Disponible en: file:///C:/Users/Usuario/Downloads/DialnetPoliticasEducativasCondicionSocialDelMagisterioCol-2670946.pdf. Acceso en: 15 de mayo de 2015.

HELG, Aline. La educación em Colombia: 1918-1957. Bogotá: Universidad Pegagógica Nacional, 2001. Disponible en: https://books.google.com.br/books?id=_Hq3Snz6DGMC $\&$ printsec $=$ frontcover $\& \mathrm{hl}=\mathrm{pt}-\mathrm{BR} \# \mathrm{v}=$ onepage $\& \mathrm{q} \& \mathrm{f}=$ false. Acceso en: 20 de maio de 2015.

HERNÁNDEZ, Gabriela. Educación de las mujeres en Pasto: del silencio a la palabra. Revista de Educación Colombiana, Boyacá. V. 14, N. 14. Diciembre de 2011. Disponible en: file:///C:/Users/Usuario/Downloads/Dialnet-EducacionDeLasMujeresEnPasto-

3819406\%20(1).pdf. Acceso en: 5 de maio de 2015.

HERNÁNDEZ, Gabriela. Las maestras en la región sur de Colombia: siglo XIX. Revista Historia de la Educación Latinoamericana, Boyacá. V. 14, N. 8, enero-junio de 2012. Disponible en: http://www.redalyc.org/pdf/869/86925890011.pdf. Acceso en: 9 de maio de 2015.

JARAMILLO, Jaime. Decreto orgánico de Instrucción Pública Nov. 1/1870. Revista Colombiana de Educación, Bogotá. $\mathrm{N}^{\circ}$ 5. Disponible en: http://www.pedagogica.edu.co/storage/ rce/articulos/5_8docu.pdf. Acceso en: 29 de maio de 2015.

MOLANO, Alfredo y VERA, César. La política educativa y el cambio social del régimen conservador a la república liberal (1903-1930). Disponible en: http://www.academia.edu/9710003/LA_POLITICA_EDUCATIVA_Y_EL_CAMBIO_SOCI AL_DEL_REGIMEN_CONSERVADOR_A_LA_REPUBLICA_LIBERAL_1903_1930. Acceso en: 12 de maio de 2015. 
OSPINA CRUZ, Carlos. De la inspección a la depresión: institutores y reformas educativas. Antioquia (1903-1930). Historia de la educación anuario, Cuidad Autónoma de Buenos Aires, v. 14, n. 1, junio de 2013. Disponible en: http://www.scielo.org.ar/scielo.php?script=sci_ arttex\&pid=S2313-92772013000100005 \&Ing=es\&nrm=iso. Acceso en: 29 de maio de 2015.

PARRA, Ana. Método lancasteriano o de enseñanza mutua en Colombia. Revista Edu-Física, Tolima. V. 11, N. 5, janeiro-junho de 2013. Disponible en: http://edu-fisica.com/Revista11/METODO-LANCASTERIANO-COLOMBIA.pdf. Acceso en: 20 de maio de 2015.

RIOS BELTRÁN, Rafael. Las ciencias de la educación en Colombia. Algunos elementos históricos sobre su apropiación e institucionalización. 1926-1954. Revista Memoria y Sociedad, Vol. 8, $\mathrm{N}^{\circ}$ 17, junio-diciembre de 2004. Departamento de Historia, Pontificia universidad Javeriana. Disponible en: http://www.javeriana.edu.co/Facultades/C_Sociales/ memoria/memoria17/rafael.pdf. Acceso en: 9 de maio de 2015.

SÁENZ, Javier. Reformas normalistas de la primera mitad del siglo XIX (1903-1946). Revista Educación y Pedagogía, Antioquia. V. 7, N ${ }^{\circ} 14-15$, noviembre de 1996. Disponible en: http://aprendeenlinea.udea.edu.co/revistas/index.php/revistaeyp/article/viewFile/5585/5007.

Acceso en: 5 de maio de 2015.

URIBE, Antonio. Revista de Instrucción Pública, Bogotá: Imprenta Nacional. 1927. Disponible en: http://www.banrepcultural.org/sites/default/files/86701/brblaa499452.pdf. Acceso en: 2 de maio de 2015.

ZULUAGA, Olga. Las escuelas normales en Colombia (durante las reformas de Francisco de Paula Santander y MARIANO Ospina Rodríguez). Historia del saber pedagógico. Revista Educación y Pedagogía, Antioquia. V. 6, N. 12 y 13, 1996. Disponible en: http://aprendeenlinea.udea.edu.co/revistas/index.php/revistaeyp/article/domnload.Acceso en: 9 de maio de 2015.

\section{Leyes}

COLOMBIA. Ley 6 de agosto de 1821. Sobre el establecimiento de las escuelas de primeras letras para niños de ambos sexos. Leyes de 1821. Colombia. Disponível em: http://www.bdigital.unal.edu.co/21/34/leyes_de_1821.pdf. Acesso em: 6 de maio de 2015.

COLOMBIA. Ley 491 de 3 de junio de 1904. Por el cual se reglamenta la ley 39 de 1903 sobre Instrucción Pública. Disponible en: http://www.mineducacion.gov.co/1621/articles102515_archivo_pdf.pdf. Acceso en: 5 de mayo de 2015.

COLOMBIA. Decreto Orgánico de Instrucción Pública de 1 de noviembre de 1870. Disponible en: http://www.pedagogica.edu.co/storage/rce/articulos/5_8docu.pdf. Acceso en: 2 de mayo de 2015.

COLOMBIA. Ley General de Educación de 8 de febrero de 1994. Disponible en: http://www.oei.es/quipu/colombia/Ley_115_1994.pdf. Acceso en: 7 de mayo de 2015. 
COLOMBIA. Constitución Pública de 1991. Disponible en: http://pdba.georgetown.edu/ Constitutions/ Colombia/colombia91.pdf. Acceso en: 10 de mayo de 2015.

COLOMBIA. Decreto Federal número 356 de 27 de agosto de 1842. Sobre el establecimiento de Escuelas Normales de mujeres. Leyes Expedidas por la Asamblea Legislativa del Estado Soberano de Panamá. Bogotá: J. F. Santillana, Imprenta del Gobierno del Estano, 1867. Disponible en: http://www.books?id=NjUrAQAAMAAJ\&printsec=frontcover\&hl=ptBR\&source $=$ gbs_ge_summary_r $\&$ cad $=0 \# v=$ onepage $\& q=1 d e c r e t o \% 20356 \& f=$ false. Acceso en: 24 e mayo de 2015. 